\title{
WordnetAffectBR: uma base lexical de palavras de emoções para a língua portuguesa *
}

\author{
Paulo Roberto Pasqualotti \\ Feevale - Instituto de Ciências Exatas e Tecnológicas - Novo Hamburgo - RS \\ ppasqualotti@gmail.com \\ Renata Vieira \\ Faculdade de Informática - PUC/RS - Porto Alegre - RS \\ renata.vieira@gmail.com
}

Resumo. Este artigo apresenta os resultados de um trabalho que integrou as áreas da Computação Afetiva e do Processamento da Linguagem Natural (PLN) no desenvolvimento de uma base de palavras de emoções para uso como um recurso computacional. Uma teoria de emoções foi utilizada como embasamento para identificar as palavras que tenham significado no domínio das emoções, gerando uma base lexical usada como referência. A partir disso, foram estudados e utilizados outros recursos que permitissem estender essa base, aumentando a cobertura do domínio das emoções com o máximo possível de palavras. Por fim, um trabalho de tradução e validação foi realizado, resultando na WordnetAffectBR, uma base de palavras de emoções para o português.

Palavras-chave: Wordnet, léxico, emoções, computação afetiva

\section{WordnetAffectBR: a lexical base of the emotion'words for Portuguese}

Abstract. This article shows the works'results that integrated Affective Computing and Natural Language Processing (NLP) areas in the development of the base emotions'words as a computing resource. An emotions'theory was used as a basement to identify words that have the emotions'domain meanings, generating a lexical foundation used as reference. Thus, it was studied and used others resources that would allow an improvement of this base, increasing emotions'domain covering with a greater number of words. Finally, a validation and translation work was accomplished, resulting in the WordNeetAffectBR, a emotions'words base for Portuguese.

Key-words: Wordnet, lexicon, emotions, affective computation

\section{Introdução}

O uso cada vez mais intenso do computador nas formas de comunicação e interação entre as pessoas tem levado a estudos no ramo da Inteligência Artificial (IA) para permitir a máquinas e sistemas computacionais reconhecer e expressar emoções. $\mathrm{O}$ campo da IA que pesquisa emoções em computadores é chamado de Computação Afetiva ("Affective Computing" em inglês) e está dividido em dois principais campos (Jaques e Vicari, 2005): (1) Emoções na Interação Homem-Computador e (2) na Síntese de Emoções.

\footnotetext{
* A base WordnetAffectBR,é parte integrante de trabalho de mestrado desenvolvido no Programa de Computação Aplicada da Unisinos/RS com orientação da professora Renata Vieira, apresentado em banca final no dia 29/02/2008.
} 
O primeiro estuda mecanismos para reconhecer e expressar emoções por máquinas na interação homem-computador (IHC). O segundo ramo investiga a simulação de emoções por máquinas (síntese de emoções) a fim de descobrir mais sobre as emoções humanas e também construir robôs que pareçam mais reais, com características mais próximas da realidade. O trabalho desenvolvido foca o primeiro ramo, mais especificamente no reconhecimento de expressões de emoções no discurso.

Atualmente, existem diversas formas de reconhecimento de emoções do usuário por meio de padrões, podendo ser citadas como as principais o reconhecimento de expressões faciais, comportamentos observáveis, entonação vocal e sinais fisiológicos (Picard, 1997). Outros trabalhos apontam para a análise automática de estados afetivos a partir de documentos escritos em linguagem natural, do inglês "automatic sentiment analysis", baseados em categorias de emoção, por exemplo, nas teorias dimensionais, nos eixos "positivo" ou "negativo" de uma emoção (Gregory et al, 2006).

Diversos trabalhos na área da análise de linguagem natural apontam para um objetivo comum, o de classificar de forma automática os aspectos subjetivos do discurso (textos ou diálogos), tais como opiniões, afetividade e emoções. Exemplos de tais trabalhos são (Genereux and Evans, 2006; Liu et al., 2003; Ma et al., 2005; Prendinger and Ishizuka, 2001), entre outros.

Estes estudam apontam para a união das áreas da Computação Afetiva e do Processamento da Linguagem Natural (PLN) no desenvolvimento de métodos, técnicas e recursos que, integrados, tornam sistemas computacionais capazes de manipular significado afetivo e de sentimentos no discurso.

Dessa integração surgem os léxicos especializados em termos afetivos que dão suporte ao desenvolvimento de trabalhos nessas áreas. Atualmente não existem ainda recursos desse tipo para a língua portuguesa. Dessa forma, o trabalho teve como objetivo suprir esta lacuna e desenvolver a primeira base de palavras de emoções para a língua portuguesa, utilizando-a em uma ferramenta de chat, como uma plataforma para os testes da base lexical, com o objetivo principal de validar sua aplicabilidade.

O desenvolvimento do trabalho explorou técnicas propostas pela computação afetiva, que é de representar visualmente uma forma textual por meio de imagens. $\mathrm{O}$ modelo proposto para a ferramenta permite que as palavras de emoções reconhecidas nas mensagens dos usuários sejam representadas como imagens na interface do chat.

A seguir é dada uma visão geral dos passos que permitiram a construção da base WordnetAffectBR: a técnica e recursos para a definição das palavras, a tradução e validação para a língua portuguesa, o modelo da ferramenta desenvolvida, assim como uma breve análise das possibilidades de uso da base.

\section{Um modelo de emoções}

O modelo OCC, definido por Ortony e colegas (Ortony et al, 1988) é um modelo psicológico cognitivo de emoções que apresenta como surgem as emoções a partir da descrição de processos cognitivos.

Esse modelo, assim chamado pela combinação das letras iniciais de seus autores, Ortony, Clore e Colins, está estruturado em 22 tipos de emoções. Neles, as palavras que identificam as emoções são: "happy for", "resentment", "gloating”, "pity”, “joy”, "distress", "pride", "shame", "admiration", "reproach", "love", "hate", "hope", "fear", "satisfaction", "fears-confirmed", "relief", "disappointment", "gratification", "remorse", "gratitude" e "anger". 
O modelo OCC foi utilizado para a constituição inicial das palavras da base de emoções, conforme listadas acima. Usada como uma referência lexical, a partir dessa estrutura foi definido o modelo e a constituição da base WordnetAffectBR.

\section{Bases lexicais}

De acordo com Jurafsky (2000), um léxico pode ser entendido como uma estrutura altamente sistemática que define o significado das palavras e como elas podem ser usadas. Para Specia e Nunes (2004), os léxicos computacionais são recursos criados, geralmente, de forma manual, especificamente para o tratamento computacional.

Diversos autores, Liu e colegas (2003), Ma e colegas (2005) entre outros, apresentaram o uso de um léxico em trabalhos de reconhecimento de emoções baseadas em texto. Ortony e colegas (1987) afirmam que o léxico afetivo permite utilizar não somente palavras que se referem diretamente a emoções, mas a muitas outras palavras, que mesmo não se referindo a emoções, implicam em diversas outras formas delas.

A base WordnetAffectBr apresenta-se como um recurso na forma de um léxico, composta de palavras pertencente a um domínio afetivo. Sua construção foi possível a partir da utilização de outros recursos lexicais: a Wordnet, a Base Affect e a Wordnet Affect.

\subsection{A base Wordnet}

A Wordnet é uma base de dados disponível de forma eletrônica, considerada como um importante recurso da área da lingüística. Criada em 1984 como um experimento linguiístico com o objetivo de ser uma base lexical da língua inglesa, desenvolvida na Universidade de Princepton $^{1}$ (Miller, 1995; Fellbaum, 1998). Atualmente conta com mais de 200.000 palavras, entre as categorias lexicais: substantivos, verbos, adjetivos e advérbios.

Essa base de dados é composta de palavras e synsets. Um synset, por sua vez, é um conjunto de sinônimos que compõem a estrutura básica da Wordnet e são descritos por um glossário. A organização dessas palavras é baseada no seu significado e forma, sendo, por isso, considerada uma "matriz lexical" bi-dimensional quanto às suas relações (Valitutti et al, 2004a).

$\mathrm{Na}$ estrutura hierárquica da Wordnet as relações ocorrem de duas formas: (1) entre as palavras, denominadas de relações lexicais e podem ser classificadas em sinonímia (mesmo significado) e antonímia (significados opostos); (2) entre os synsets, denominadas de relações semânticas, ou semântico-conceituais, tendo como principais relações a hipernímia e hiponímia:(entre as classes, por exemplo, "mamífero" é uma superclasse de "cachorro") e meronímia e holonímia (entre as partes e o todo, por exemplo, árvore é membro de floresta, assim como hidrogênio é substância de água).

\subsection{As bases afetivas}

Devido à necessidade de relacionar o conteúdo lexical e semântico das palavras da Wordnet com significado afetivo, foram utilizados outros recursos que, pelas características e conteúdos, possibilitassem atender à demanda da pesquisa em estender a base das palavras de emoções.

A base Affect é um recurso lingüístico desenvolvido pelos pesquisadores do

grupo $\mathrm{TCC}^{2}$, criado manualmente. Sua parte principal é composta pelas classes gramaticais da Wordnet e outras informações lexicais, semânticas e afetivas.

As informações lexicais e semânticas referem-se à correlação entre as línguas inglesa e italiana, à classe gramatical a qual a palavra pertence, às relações de sinonímia e antonímia, e também um glossário com uma breve descrição. As informações afetivas, 
por sua vez, referem-se às teorias de emoções baseadas no conceito da avaliação cognitiva (Ortony et al., 1987), às teorias das emoções básicas (Elliot, 1992) e às teorias dimensionais, representando sua valência afetiva: positivo ou negativo.

A partir das informações afetivas da base Affect, os autores focaram na criação de outra base que tivesse somente conteúdo relacionado aos estados afetivos e que pudesse ser utilizada com um recurso no domínio da afetividade.

Para o desenvolvimento dessa base, os trabalhos foram divididos em dois estágios distintos (Valitutti et al, 2004b):

O primeiro consistiu da projeção dos termos da base Affect para os respectivos synsets na base Wordnet Affect. A informação afetiva da base Affect foi acrescentada aos synsets correspondentes da Wordnet Affect, com uma etiqueta afetiva, denominada de "a-label", conforme Tabela 1. A informação projetada nos "a-labels" é o conteúdo da informação da teoria cognitiva da base Affect.

Tabela 1 - Lista de "a-labels" com os respectivos estados afetivos e exemplos.

\begin{tabular}{|l|l|l|}
\hline a-label & Estado afetivo & Exemplos \\
\hline Emo & emotion & substantivo "anger", verbo "fear" \\
\hline Môo & mood & substantivo "animosity", adjetivo "amiable" \\
\hline Tra & Trait & substantivo "aggressiveness \\
\hline Cog & cognitive state & substantivo "confusion", adjetivo "dazed" \\
\hline Phy & physical state & substantivo "illness", adjetivo "all_in" \\
\hline Eds & edonic signal & substantivo "hurt", substantivo "suffering" \\
\hline Sit & emotion- situation eliciting & substantivo "awkwardness \\
\hline Res & emotional response & substantivo "cold_sweat", verbo "tremble" \\
\hline Beh & behaviour & substantivo "offense", adjetivo "inhibited" \\
\hline Att & attitude & substantivo "intolerance", noun "defensive" \\
\hline Sen & sensation & substantivo "coldness", verbo "feel" \\
\hline
\end{tabular}

Numa segunda etapa foram realizadas as correlações com os synsets da Wordnet, onde relações semânticas foram utilizadas para alimentar a Wordnet Affect. A cada relação semântica da Wordnet (antônimo, similaridade, "pertencer a", atributos, "vertambém" e "derivado-de"), foi verificado se o significado afetivo era preservado. As relações de hiperônimo, implicação, causas e grupos-de-verbos, onde o significado afetivo é parcialmente preservado, não foram consideradas para compor a base afetiva proposta.

A Tabela 2 apresenta alguns registros da base Wordnet Affect. A coluna "CAT" é a etiqueta ("a-label") que contém a informação afetiva da base Affect.

Tabela 2 - Estrutura da Wordnet Affect e exemplos de registros da base

\begin{tabular}{|l|l|l|l|}
\hline POS & ID do Synset & Origem & CAT \\
\hline $\mathrm{N}$ & $\# 10972097$ & core & emo \\
\hline $\mathrm{N}$ & $\# 03848510$ & core & beh, att, tra \\
\hline $\mathrm{V}$ & $\# 00548199$ & antonym & emo, cog \\
\hline $\mathrm{A}$ & $\# 02475653$ & pertains-to & eds,emo \\
\hline
\end{tabular}

Os valores para os campos da Wordnet Affect, descritos na Tabela 2 são:

a) POS ("Part Of Speech"): a classe gramatical do synset; 
b) ID do SYNSET: código de localização do synset na base Wordnet;

c) ORIGEM: forma como o synset foi obtido. Da base Affect ("core") ou das relações da Wordnet;

d) CAT: é a "etiqueta afetiva" do synset, baseado em Ortony e colegas (1987).

\section{Construção da base WordnetAffectBR}

Duas premissas foram fundamentais na construção da base proposta: que os synsets identificados na Wordnet tivessem uma relação com o dado "ID do synset" da base Wordnet Affect e com o estado afetivo das emoções, identificadas no campo "CAT" da base como "Emo".

As etapas para construção da base WordnetAffectBR, apresentadas na Figura 2, são detalhadas resumidamente abaixo:

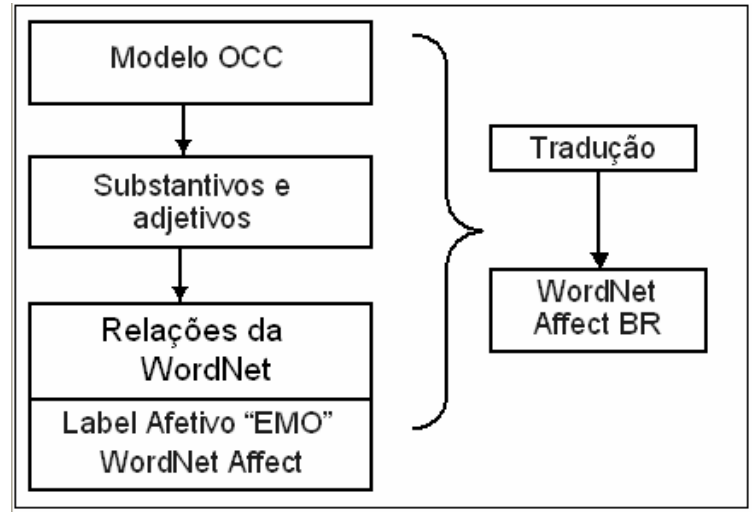

Figura 2: Estrutura do trabalho de criação da base Wordnet Affect BR.

a) as palavras de emoções do Modelo OCC3 formaram a referência lexical para o trabalho com 21 palavras;

b) uma base, considerando substantivos e respectivos adjetivos, do modelo OCC foi criada contendo 42 palavras;

c) cada uma das palavras foi processada a partir das relações semânticas e lexicais das bases Wordnet e as etiquetas afetivas da Wordnet Affect. Nessa fase foram consideradas adequadas as relações de sinonímia, similaridade e "ver também..." para adjetivos e sinonímia, hipernímia e hiponímia para substantivos.

Como resultado da fase de extensão da lista de palavras de emoções, uma nova base foi obtida, totalizando 403 palavras.

\subsection{Tradução e validação das palavras}

A tradução para o português foi realizada por tradutoras selecionadas a partir de entrevistas, ambas com experiências em trabalhos dessa natureza e com vivência em países da língua inglesa, com boa fluência e conhecimento dessa língua.

A orientação que norteou todo o processo foi de que a tradução não deveria contemplar somente o sentido isolado das palavras, mas sim considerar o seu significado presente nas definições da Wordnet e na estrutura dos synsets. Por isso, além das palavras, o glossário de cada synset também foi traduzido.

A compreensão e entendimento pelas tradutoras do significado presente em cada synset foram fundamentais para a atividade de tradução, pois na Wordnet uma palavra pode fazer parte de mais de um synset, com significados diferentes.

A metodologia de tradução foi dividir a base entre as tradutoras, num primeiro momento, onde a palavra e o glossário foram traduzidos. Num segundo momento, cada uma traduziu a segunda parte, num processo inverso. Após, foi realizada a validação por 
meio de um processamento, cruzando as palavras traduzidas em cada fase. Essas bases foram listadas e avaliadas quanto à diferença de conteúdo, numa análise e discussão das situações que apresentaram diferenças no seu resultado. Essa etapa permitiu a correção e a escolha da palavra mais adequada para a tradução.

Foram identificadas que as diferenças entre as traduções geraram relações de sinonímia, permitindo, dessa forma, incrementar a base de palavras. Em algumas situações, quando havia divergência entre as traduções, o consenso foi por uma das palavras, descartando a outra.

As fases de tradução e validação das palavras de emoção para a língua portuguesa apresentaram algumas ocorrências de duplicidades e de divergências quanto à sua aplicação, cujos detalhes são relatados a seguir:

a) Como exemplo de duplicidades, a emoção "relief" possui sete sentidos na Wordnet. Dessas, quatro foram traduzidas como "alívio". Para a composição final da base, as palavras duplicadas foram desconsideradas.

b) Quanto à aplicação na língua portuguesa, algumas situações exigiram uma maior discussão e estudo para que a tradução mantivesse o escopo dado no trabalho, ou seja, utilizar palavras com significado de emoção. Por exemplo, a emoção "gloating", que, de acordo com o modelo OCC, indica satisfação por um evento indesejável para outra pessoa, não possui uma tradução adequada para uma palavra da língua portuguesa. A tradução resultou na expressão "prazer pelo sofrimento alheio" e "alegria pelo sofrimento alheio", mantendo, dessa forma, o sentido, mas com uma diferença na sua estrutura, pois a palavra em inglês é traduzida na língua portuguesa para uma expressão mais complexa, no caso, uma frase.

Dessa forma, a base original da WordnetAffectBR, que, na fase final da tradução apresentou 457 palavras, após a retirada das duplicidades e análise dos significados, ficou composta de 289 palavras.

\section{Ferramenta de chat}

Para aplicação da base afetiva, foi desenvolvida uma ferramenta de Chat que reconhece palavras de emoção presentes no diálogo e representa-as na tela por meio de imagens.

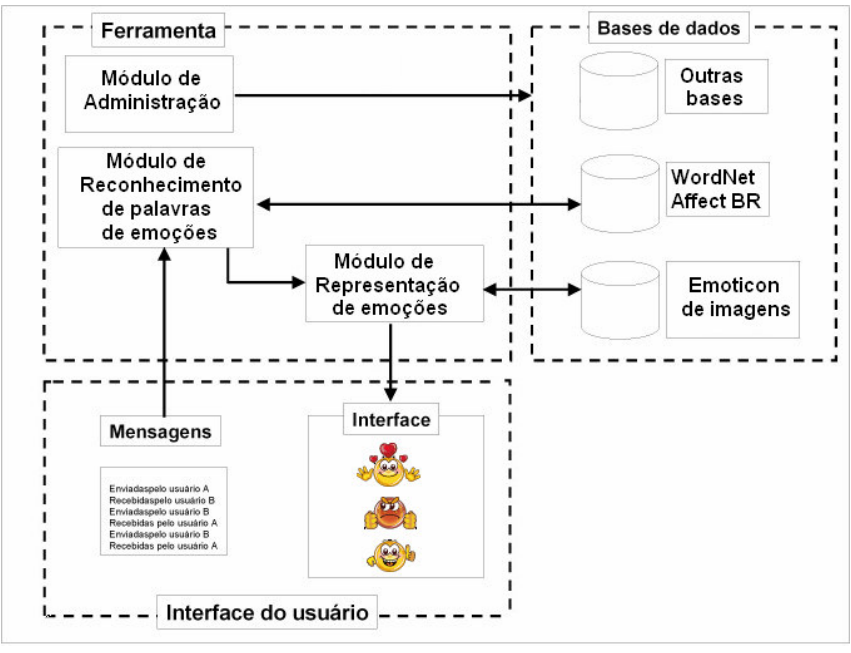

Figura 3 - Modelo da ferramenta

Para a representação visual da palavra de emoção reconhecida no diálogo, o algoritmo utiliza os dados e relacionamentos entre as tabelas das bases de palavras, 
retornando para o módulo de interface do usuário a identificação da imagem que deve ser apresentada na tela.

A Figura 3 apresenta o modelo da ferramenta, destacando o fluxo da mensagem e das ações dos módulos de reconhecimento e representação das imagens.

A mensagem enviada pelos usuários é tratada pelo módulo de reconhecimento, permitindo que se possam identificar as ocorrências das palavras de emoções na mensagem. Elas são enviadas pelos usuários e tratadas como uma expressão completa, ou seja, um conjunto de palavras separadas por espaços em branco, podendo conter sinais de pontuação e apresentar palavras maiúsculas ou minúsculas.

As mensagens são apresentadas aos usuários salientando as palavras de emoções em negrito e a respectiva imagem, como pode ser observado na Figura 4.

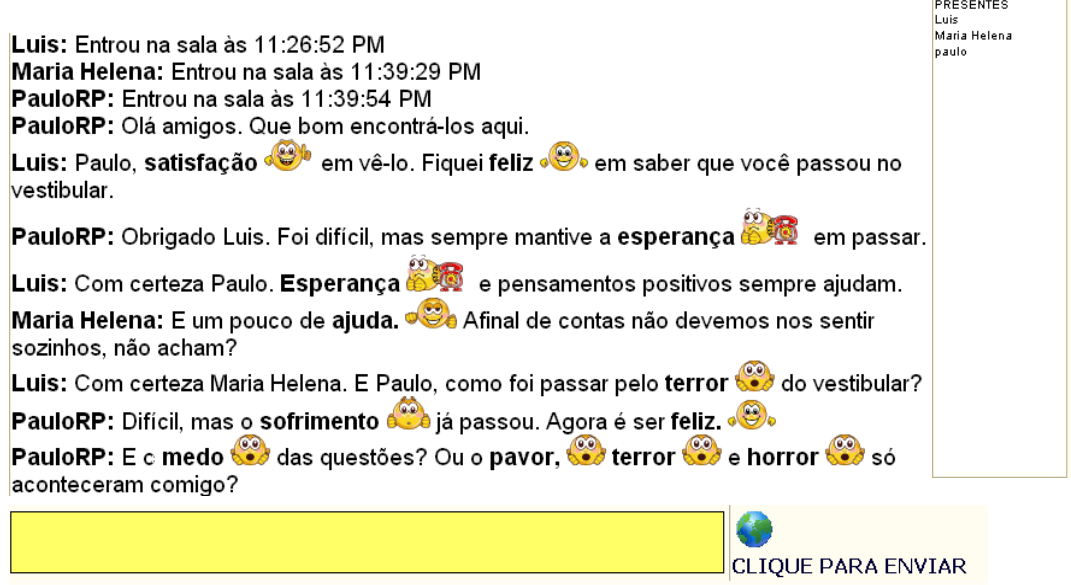

Figura 4 - Interface da tela da ferramenta de chat

\section{Considerações finais}

Dotar sistemas computacionais com a capacidade de perceber e representar significados de emoções é fundamental para a comunicação e o relacionamento entre as pessoas, pois é no entendimento dos significados, muitas vezes subjetivos do diálogo, que a interação se desenvolve. Portanto, ao pensar em sistemas computacionais para a comunicação on-line, cujo papel seja o da mediação do diálogo, deve-se pensar e projetá-los com a capacidade de darem o devido tratamento à presença de emoções nas reações e ações dos usuários.

Para isso é necessário que haja o entendimento de como os sujeitos se comportam diante de determinada situação e quais as formas e intensidades nas quais as emoções se apresentam. Dessa forma será possível desenvolver interfaces que representem emoções, bem como criar sistemas computacionais que reconheçam esses estados emocionais, semelhante à forma como ocorre com as pessoas durante uma interação. Dessa forma a base lexical desenvolvida neste trabalho pode ser utilizada em trabalhos que têm o objetivo de reconhecer palavras de emoções em recursos baseados em texto, assim como representá-las por meio de imagens.

A base WordnetAffectBR pode ser usada no desenvolvimento de outras aplicações que façam uso de um léxico afetivo, seja quanto à sua ocorrência, como realizado no presente trabalho, ou em trabalhos que busquem inferir quanto ao estado emocional das pessoas. Essa base foi desenvolvida a partir de uma teoria que apresenta as emoções, bem como justifica as formas de surgimento de cada uma delas pela 
capacidade cognitiva das pessoas em interpretar significados diante da sua própria interpretação de mundo. Da mesma forma, a metodologia utilizada para estender a abrangência da base de palavras utiliza a Wordnet e a Wordnet Affect, recursos lexicais amplamente validados e utilizados pelas áreas da Computação Afetiva e o PLN.

A ferramenta de Chat utiliza a base WordnetAffectBR, agregando recursos adicionais àqueles usualmente encontrados em programas de comunicação síncrona. Além de permitir aos usuários enviar e receber mensagens, a ferramenta incorpora capacidades de reconhecimento e representações de palavras de emoção numa forma visual, com imagens que transmitem e representam significados.

Os experimentos realizados durante a pesquisa e o desenvolvimento do presente trabalho, apontam para diversas outras possibilidades, tanto de funcionalidades da ferramenta quanto da abrangência da base WordnetAffectBR.

Quanto à base, podem ser destacados para implementação futura o tratamento de formas e tempos verbais, palavras derivadas, de gênero e número, palavras compostas, uso de expressões regionais, gírias e metáforas, expressões na forma de "emoticons", além de incluir o tratamento de outros estados afetivos, baseados nas etiquetas afetivas da WordNet Affect, tais como: traços de personalidade, humor, comportamento, entre outros.

Em aplicações e sistemas computacionais, foram identificadas no trabalho possibilidade para uso da Língua Brasileira de Sinais (LIBRAS), agregar sistemas de correção automática das mensagens, análise do nível de satisfação de clientes, cálculo da valência ${ }^{4}$ em processamento de textos, entre outros.

O trabalho apresentado foi o primeiro passo para o desenvolvimento de trabalhos relacionados à subjetividade e afetividade no discurso da Língua Portuguesa. A base lexical WordnetAffectBR é um recurso que pode ser expandido à medida que novas implementações surgirem. Essa base poderá servir para outros propósitos de estudos e pesquisas envolvendo o uso de um léxico de emoções, estando disponível para propósitos dessa natureza.

\section{Notas}

1 Atualmente a Wordnet está na sua versão 2.1 liberada em março de 2005 para Windows e na versão 3.0, liberada em dezembro de 2006 para Unix, Linux e Solaris.

2 A divisão de pesquisa TCC ("The cognitive and Communication Technologies") integra o ITC-Irst, um grupo de pesquisa europeu das áreas do Processamento da Linguagem Natural e da Computação. Disponível em http://tcc.itc.it/

${ }^{3} \mathrm{O}$ modelo OCC apresenta 22 emoções. Para este trabalho foram consideradas 21, pois as emoções "fear" e "fears confirmed" foram agrupadas em uma única emoção definida como "fear".

${ }^{4}$ Emoções são reações com valência (positiva ou negativa) baseadas na situação que as dispara, a partir da avaliação cognitiva e psicológica que o sujeito faz diante das conseqüências dos eventos, das ações de agentes e da atratividade exercida pelos objetos (Ortony et al, 1987).

\section{Referências Bibliográficas}

Elliot, C. D. The Affective Reasoner: a process model of emotions in a multi-agent system. Ph.D. thesis, Northwestern University, Evanston, Illinois, 1992.

Fellbaum, C. Wordnet: an electronic lexical database. MIT Press. 1998 
Genereux, Michel; Evans, Roger. Towards a validated model for affective classification of texts Proceedings of the Workshop on Sentiment and Subjectivity in Text, pages 55-62, Sydney, July 2006. c 2006 Association for Computational Linguistics.

Gregory, Michelle L.;Chinchor, Nancy;Whitney, Paul; Carter, Richard; Hetzler,Elizabeth; Turner, Alan. User-directed Sentiment Analysis: Visualizing the Affective Content of Documents. Proceedings of the Workshop on Sentiment and Subjectivity in Text, pages 23-30, Sydney, July 2006. c2006 Association for Computational Linguistics

Jaques, Patrícia Augustin. Vicari, Rosa Maria. Estado da arte em ambientes inteligentes de aprendizagem que consideram a afetividade do aluno. Artigo publicado na revista Informática na Educação: Teoria \& Prática, vol.8, número 1, 2005. Disponível em http://www.pgie.ufrgs.br/revista)

Jurafsky, D.; Martin, J. Speech and Language Processing: an Introduction to Natural Language Processing, Computational Linguistics and Speech Recognition, Upper Saddle. 2000. River, New Jersey, Prentice Hall. (pp. 1-18)

Liu. Hugo, Lieberman Henry, Selker Ted. A model of textual affect sensing using real-world knowledge, Proceedings of the 8th international conference on Intelligent user interfaces, January 12-15, 2003, Miami, Florida, USA

Ma, Chunling. Prendinger, Helmut. Ishizuka, Mitsuru. Emotion Estimation and Reasoning Based on Affective Textual Interaction, in Affective Computing and Intelligent Interaction (First Int'l Conf. ACII 2005) (J. Tao, T. Tan and R. W. Picard (Eds.)), Springer LNCS 3784, Beijing, China, pp.622-628 (2005.10)

Ma, Chunling. Prendinger, Helmut. Ishizuka, Mitsuru. A Chat System Based on Emotion Estimation from Text and Embodied Conversational Messengers, in Entertaiment Computing -- ICEC 2005 (Proc. IFIP 4th Int'l Conf. on Entertainment Computing-2005, Kobe-Sanda, Japan), Springer LNCS 3711, pp.535-538 (2005.9)

Miller, A.G. Wordnet: A Lexical Database for English, Communications of the ACM, Vol. 38, No.11, November 1995, pp. 39 - 41.

Ortony, A.; Clore, G.L.; Foss, M.A. The Referential Structure of the Affective Lexicon. Universiry of Illinois at Urbana-Champaign (1987)

Ortony, A.; Clore, G.L.; Colins, A. The Cognitive Structure of Emotions, Cambridge University Press. 1988

Picard, Rosling. Affective computing. Cambridge, Massachustes Institure of Technology. The MIT Press, 1997.

Prendinger, Helmut; Ishizuka, Mitsuru. Simulating Affective Communication with Animated Agents Proc. Eighth IFIP TC.13 Conference on Human-Computer Interaction (INTERACT 2001), pp.182-189, Tokyo, Japan (2001.7).

Helmut Prendinger and Mitsuru Ishizuka: Affective and Social Behavior in Animated Agents (Invited Paper), CD-Rom Proc. 9th IFSA World Congress and 20th NAFIPS Int'l Conf., Session on Kansei Systems, pp.2918-2923, Vancouver, Canada (2001.7).

Specia, Lucia. Nunes, Maria das Graças Volpe. Desambiguação Lexical Automática de Sentido: Um Panorama. Série de Relatórios do Núcleo Interinstitucional de 
Lingüística Computacional. NILC - ICMC-USP, Caixa Postal 668, 13560-970 São Carlos, SP, Brasil, 2004

Valitutti, Alessandro, Strapparava, Carlo e, Sttock, Oliveiro. Developing Affective Lexical Resources. PsychNology Journal, 2004 Volume 2, Number 1, 61 - 83

Valitutti, Alessandro, Strapparava, Carlo. Wordnet-Affect: an affective extension of Wordnet. In Proceedings ofthe 4th International Conference on Language Resources and Evaluation (LREC 2004), Lisbon, May 2004, pp. 1083-1086. 